Irish Math. Soc. Bulletin

Number 73, Summer 2014, 21-28

ISSN 0791-5578

\title{
TWO TRIGONOMETRIC IDENTITIES
}

\author{
HORST ALZER AND WENCHANG CHU
}

Abstract. We show that the trigonometric identities

$$
\prod_{k=1}^{n-1}\left\{1-\cos \frac{2 k \pi}{n}\right\}^{(n-k) \ell+m}=2^{(1-n)(\ell n / 2+m)} n^{\ell n+2 m}
$$

and

$$
\prod_{k=1}^{n-1}\left\{\cos (2 \theta)-\cos \frac{2 k \pi}{n}\right\}^{(n-k) \ell+m}=2^{(1-n)(\ell n / 2+m)}\left\{\frac{\sin (n \theta)}{\sin \theta}\right\}^{\ell n+2 m}
$$

are valid for all $\ell, m \in \mathbf{Z}$ and $2 \leq n \in \mathbf{N}$. They extend the results due to Baica and Gregorac, who proved the identities for the special case $\ell=1, m=-1$. Moreover, we determine all $\ell, m, n$ such that the first trigonometric product just displayed is an integer.

In 1986, Baica [1] applied methods from cyclotomic fields to provide a rather long and complicated proof for the following interesting trigonometric identity:

$$
\prod_{k=1}^{n-1}\left\{1-\cos \frac{2 k \pi}{n}\right\}^{n-k-1}=2^{(1-n)(n / 2-1)} n^{n-2}
$$

where $n=2,3,4, \cdots$. Baica also remarked that "any proof avoiding cyclotomic fields could be very difficult, if not insoluble" [1, P. 705].

In 1989, Gregorac [3] used properties of Chebyshev polynomials to present a new proof of (1). Actually, he proved the identity

$$
\prod_{k=1}^{n-1}\left\{\cos (2 \theta)-\cos \frac{2 k \pi}{n}\right\}^{n-k-1}=2^{(1-n)(n / 2-1)}\left\{\frac{\sin (n \theta)}{\sin \theta}\right\}^{n-2}
$$

for $n=2,3,4, \cdots$, which, letting $\theta$ tend to 0 , leads to (1).

2010 Mathematics Subject Classification. 26A09, 33B10.

Key words and phrases. Trigonometric identity, finite product, sharp bound, divisibility.

Received on 9-9-2013; revised 16-3-2014. 
Here, we extend (1) and (2). First, we offer an elementary short and simple proof of a generalization of Baica's identity. In order to verify our result we only make use of three well-known properties of sine and cosine,

$$
\begin{aligned}
& 1-\cos (2 \theta)=2 \sin ^{2} \theta \\
& \sin (\pi-\theta)=\sin \theta \\
& \prod_{k=1}^{n-1} \sin \frac{k \pi}{n}=2^{1-n} n
\end{aligned}
$$

Formula (5) as well as many related formulas involving trigonometric functions can be found in [2, Eq. 4.14].

We have the following extension of identity (1).

Theorem 1. Let $\ell, m$ be integers and let $n \geq 2$ be a natural number. Then,

$$
\prod_{k=1}^{n-1}\left\{1-\cos \frac{2 k \pi}{n}\right\}^{(n-k) \ell+m}=2^{(1-n)(\ell n / 2+m)} n^{\ell n+2 m} .
$$

Proof. Applying (3) yields

$$
\prod_{k=1}^{n-1}\left\{1-\cos \frac{2 k \pi}{n}\right\}^{(n-k) \ell+m}=2^{(n-1)(\ell n / 2+m)} \prod_{k=1}^{n-1}\left\{\sin \frac{k \pi}{n}\right\}^{2[(n-k) \ell+m]} .
$$

From (4) we conclude that

$$
\prod_{k=1}^{n-1}\left\{\sin \frac{k \pi}{n}\right\}^{(n-k) \ell+m}=\prod_{k=1}^{n-1}\left\{\sin \frac{(n-k) \pi}{n}\right\}^{k \ell+m}=\prod_{k=1}^{n-1}\left\{\sin \frac{k \pi}{n}\right\}^{k \ell+m}
$$


Using (8) and (5) gives

$$
\begin{aligned}
\prod_{k=1}^{n-1}\left\{\sin \frac{k \pi}{n}\right\}^{2[(n-k) \ell+m]} & =\prod_{k=1}^{n-1}\left\{\sin \frac{k \pi}{n}\right\}^{(n-k) \ell+m} \prod_{k=1}^{n-1}\left\{\sin \frac{k \pi}{n}\right\}^{(n-k) \ell+m} \\
& =\prod_{k=1}^{n-1}\left\{\sin \frac{k \pi}{n}\right\}^{(n-k) \ell+m} \prod_{k=1}^{n-1}\left\{\sin \frac{k \pi}{n}\right\}^{k \ell+m} \\
& =\prod_{k=1}^{n-1}\left\{\sin \frac{k \pi}{n}\right\}^{\ell n+2 m}=\left(2^{1-n} n\right)^{\ell n+2 m}
\end{aligned}
$$

Combining (7) and (9) leads to (6).

Next, we extend Gregorac's identity (2). We need the following formulas:

$$
\begin{gathered}
\sin \left(\frac{\pi}{2}-\theta\right)=\cos \theta, \\
\sin (2 \theta)=2 \sin \theta \cos \theta, \\
\cos y-\cos x=2 \sin \frac{x+y}{2} \sin \frac{x-y}{2}, \\
\frac{\sin (n \theta)}{\sin \theta}=2^{n-1} \prod_{k=1}^{n-1}\left\{\cos \theta-\cos \frac{k \pi}{n}\right\} .
\end{gathered}
$$

Identity (13) is the well-known product representation for the Chebyshev polynomials of the second kind.

Theorem 2. Let $\ell, m$ be integers and let $n \geq 2$ be a natural number. Then, for $\theta \in \mathbf{R}$,

$$
\prod_{k=1}^{n-1}\left\{\cos (2 \theta)-\cos \frac{2 k \pi}{n}\right\}^{(n-k) \ell+m}=2^{(1-n)(\ell n / 2+m)}\left\{\frac{\sin (n \theta)}{\sin \theta}\right\}^{\ell n+2 m} .
$$

Proof. Using (10) gives

$$
\prod_{k=1}^{n-1} \sin \left(\frac{k \pi}{2 n}-\frac{\theta}{2}\right)=\prod_{k=1}^{n-1} \sin \left(\frac{(n-k) \pi}{2 n}-\frac{\theta}{2}\right)=\prod_{k=1}^{n-1} \cos \left(\frac{k \pi}{2 n}+\frac{\theta}{2}\right) .
$$


Now, we apply (12), (15) and (11). Then we have

$$
\begin{aligned}
\prod_{k=1}^{n-1}\left\{\cos \theta-\cos \frac{k \pi}{n}\right\} & =\prod_{k=1}^{n-1}\left\{2 \sin \left(\frac{k \pi}{2 n}+\frac{\theta}{2}\right) \sin \left(\frac{k \pi}{2 n}-\frac{\theta}{2}\right)\right\} \\
& =\prod_{k=1}^{n-1}\left\{2 \sin \left(\frac{k \pi}{2 n}+\frac{\theta}{2}\right) \cos \left(\frac{k \pi}{2 n}+\frac{\theta}{2}\right)\right\} \\
& =\prod_{k=1}^{n-1} \sin \left(\frac{k \pi}{n}+\theta\right)
\end{aligned}
$$

From (4) and (12) we obtain

$$
\begin{aligned}
\prod_{k=1}^{n-1} \sin ^{2}\left(\frac{k \pi}{n}+\theta\right) & =\prod_{k=1}^{n-1}\left\{\sin \left(\frac{k \pi}{n}+\theta\right) \sin \left(\frac{(n-k) \pi}{n}-\theta\right)\right\} \\
& =\prod_{k=1}^{n-1}\left\{\sin \left(\frac{k \pi}{n}+\theta\right) \sin \left(\frac{k \pi}{n}-\theta\right)\right\} \\
& =2^{1-n} \prod_{k=1}^{n-1}\left\{\cos (2 \theta)-\cos \frac{2 k \pi}{n}\right\}
\end{aligned}
$$

Applying (13), (16) and (17) yields

$$
\begin{aligned}
\left\{\frac{\sin (n \theta)}{\sin \theta}\right\}^{2 m} & =2^{2 m(n-1)} \prod_{k=1}^{n-1}\left\{\cos \theta-\cos \frac{k \pi}{n}\right\}^{2 m} \\
& =2^{2 m(n-1)} \prod_{k=1}^{n-1} \sin ^{2 m}\left(\frac{k \pi}{n}+\theta\right) \\
& =2^{m(n-1)} \prod_{k=1}^{n-1}\left\{\cos (2 \theta)-\cos \frac{2 k \pi}{n}\right\}^{m}
\end{aligned}
$$


From (4) and (12) we get

$$
\begin{aligned}
\prod_{k=1}^{n-1} \sin ^{n}\left(\frac{k \pi}{n}+\theta\right) & =\prod_{k=1}^{n-1}\left\{\sin ^{n-k}\left(\frac{k \pi}{n}+\theta\right) \sin ^{k}\left(\frac{k \pi}{n}+\theta\right)\right\} \\
& =\prod_{k=1}^{n-1}\left\{\sin ^{k}\left(\frac{(n-k) \pi}{n}+\theta\right) \sin ^{k}\left(\frac{k \pi}{n}+\theta\right)\right\} \\
& =\prod_{k=1}^{n-1}\left\{\sin ^{k}\left(\frac{k \pi}{n}-\theta\right) \sin ^{k}\left(\frac{k \pi}{n}+\theta\right)\right\} \\
& =2^{(1-n) n / 2} \prod_{k=1}^{n-1}\left\{\cos (2 \theta)-\cos \frac{2 k \pi}{n}\right\}^{k} .
\end{aligned}
$$

Combining (13), (16) and (19) gives

$$
\left\{\frac{\sin (n \theta)}{\sin \theta}\right\}^{n}=2^{(n-1) n / 2} \prod_{k=1}^{n-1}\left\{\cos (2 \theta)-\cos \frac{2 k \pi}{n}\right\}^{k} .
$$

Finally, (18) and (20) lead to

$$
\begin{aligned}
\left\{\frac{\sin (n \theta)}{\sin \theta}\right\}^{2 m+\ell n} & =2^{(n-1)(\ell n / 2+m)} \prod_{k=1}^{n-1}\left\{\cos (2 \theta)-\cos \frac{2 k \pi}{n}\right\}^{m+k \ell} \\
& =2^{(n-1)(\ell n / 2+m)} \prod_{k=1}^{n-1}\left\{\cos (2 \theta)-\cos \frac{2 k \pi}{n}\right\}^{(n-k) \ell+m} .
\end{aligned}
$$

This is equivalent to (14).

Remark 1. Setting $\ell=1$ and $m=-1$ in (6) and (14), respectively, gives (1) and (2).

Remark 2. Let $\ell, m \in \mathbf{Z}$ and $2 \leq n \in \mathbf{N}$ with $\ell n+2 m>0$. Applying (14) and the well-known inequality

$$
\left|\frac{\sin (n \theta)}{n \sin \theta}\right| \leq 1 \quad(n=1,2,3, \ldots)
$$

we obtain for all $\theta \in \mathbf{R}$ :

$$
\prod_{k=1}^{n-1}\left\{\cos (2 \theta)-\cos \frac{2 k \pi}{n}\right\}^{(n-k) \ell+m} \leq 2^{(1-n)(\ell n / 2+m)} n^{\ell n+2 m} .
$$


Setting $\theta=0$ we conclude from (6) that the given upper bound is sharp. If $\ell n+2 m<0$, then (21) holds with " $\geq$ " instead of " $\leq$ ".

The representation (6) reveals that if $\ell, m \in \mathbf{Z}, 2 \leq n \in \mathbf{N}$, then the product

$$
P_{n}(\ell, m)=\prod_{k=1}^{n-1}\left\{1-\cos \frac{2 k \pi}{n}\right\}^{(n-k) \ell+m}
$$

is a rational number. In view of this result it is natural to ask whether there exist numbers $\ell, m, n$ such that $P_{n}(\ell, m)$ is an integer. The next theorem answers this question.

Theorem 3. Let $\ell, m$ be integers and $n \geq 2$ a natural number. The product $P_{n}(\ell, m)$ is an integer if and only if $\ell n+2 m=0$

$$
\begin{array}{llll}
\text { or } \quad l n+2 m>0 & \text { with } \quad n=2^{r} \quad(r=1,2) ; \\
\text { or } \quad l n+2 m<0 & \text { with } \quad n=2^{r} \quad(3 \leq r \in \mathbf{N}) .
\end{array}
$$

Proof. Using (6) we obtain:

if $\ell n+2 m=0$, then $P_{n}(\ell, m)=1$;

if $n=2^{r}(r=1,2)$ and $\ell n+2 m>0$, then

$$
P_{n}(\ell, m)=2^{(\ell n+2 m) / 2} \in \mathbf{Z} ;
$$

if $n=2^{r}(r \geq 3)$ and $\ell n+2 m<0$, then

$$
P_{n}(\ell, m)=2^{-\left(2^{r}-2 r-1\right)(\ell n+2 m) / 2} \in \mathbf{Z} .
$$

Now, let $P_{n}(\ell, m) \in \mathbf{Z}$. We asssume (for a contradiction) that none of (22), (23) and $\ell n+2 m=0$ is satisfied. We have

$$
\begin{aligned}
& P_{2}(\ell, m)=2^{\ell+m}, \\
& P_{3}(\ell, m)=\left\{\frac{3}{2}\right\}^{3 \ell+2 m}, \\
& P_{4}(\ell, m)=2^{2 \ell+m}, \\
& P_{5}(\ell, m)=\left\{\frac{5}{4}\right\}^{5 \ell+2 m} .
\end{aligned}
$$

Case 1. $\ell n+2 m>0$.

Then, $P_{3}(\ell, m) \notin \mathbf{Z}$ and $P_{5}(\ell, m) \notin \mathbf{Z}$. Let $n \geq 6$. From

$$
2^{(n-1)(\ell n+2 m) / 2} \cdot K=n^{\ell n+2 m} \quad(K \in \mathbf{N})
$$


we conclude that 2 divides $n^{\ell n+2 m}$. This implies that $n$ is even. Let $n=2^{r} q$, where $r \geq 1$ and $q$ is odd. Then, (24) leads to

$$
2^{((n-1) / 2-r)(\ell n+2 m)} \cdot K=q^{\ell n+2 m} .
$$

Since $q$ is odd, we obtain

$$
\frac{n-1}{2}-r \leq 0
$$

Hence,

$$
2^{r} \leq 2^{r} q=n \leq 2 r+1 .
$$

If follows that $r=1$ or $r=2$. However, this contradicts (25), since $n \geq 6$.

Case 2. $\ell n+2 m<0$.

Then, $P_{n}(\ell, m) \notin \mathbf{Z}$ for $n=2,3,4,5$. Let $n \geq 6$. From (24) we obtain

$$
n^{-(\ell n+2 m)} \cdot K=2^{-(n-1)(\ell n+2 m) / 2} .
$$

This yields $n=2^{r}$ with $r \geq 3$. A contradiction. The proof is complete.

Acknowledgement. We thank the referee for a careful reading of the manuscript.

\section{REFERENCES}

[1] M. Baica, Trigonometric identities, Intern. J. Math. Math. Sci. 9 (1986), 705-714.

[2] H.W. Gould, Combinatorial identities: Table III: Binomial identities derived from trigonometric and exponential series, www.math.wvu.edu/ gould.

[3] R.J. Gregorac, On Baica's trigonometric identity, Intern. J. Math. Math. Sci. 12 (1989), 119-122.

Horst Alzer received his doctoral degree in mathematics from the University of Bonn and his habilitation in mathematics from the University of urzburg. His main interest of research are inequalities and special functions.

Wenchang Chu received, in July 1987, his $\mathrm{PhD}$ in Combinatorial mathematics under the supervision of Prof. L. C. Hsu at Dalian University of Technology (P.R.China). Since then he has been employed in Academia Sinica (Beijing), Università di Roma La Sapienza, Freie Universität Berlin, INRIA (Paris), and 
currently in Salento University (Italy). His research interests cover broadly the interaction among classical analysis, combinatorics and special functions.

(Alzer) Morsbacher Str. 10, 51545 Waldbröl, Germany

E-mail address, Alzer: H.Alzer@gmx.de

(Chu) Dipartimento di Matematica e Fisica "Ennio De Giorgi", UniVersità del Salento, Lecce-Arnesano, P. O. Box 193, 73100 Lecce, ITALY

E-mail address, Chu: chu.wenchang@unisalento.it 